\title{
Correction: Long-read trio sequencing of individuals with unsolved intellectual disability
}

Marc Pauper - Erdi Kucuk (10 - Aaron M. Wenger - Shreyasee Chakraborty • Primo Baybayan • Michael Kwint • Bart van der Sanden (1) - Marcel R. Nelen - Ronny Derks • Han G. Brunner - Alexander Hoischen •

Lisenka E. L. M. Vissers (1) - Christian Gilissen (1)

Published online: 26 March 2021

(c) The Author(s), under exclusive licence to European Society of Human Genetics 2021

Correction to: Eur J Hum Genet

https://doi.org/10.1038/s41431-020-00770-0
The authors inadvertently included tracked changes in their Supplemental Material file in error. These have been now been removed. 